\title{
Lesiones agudas de los tendones flexores
}

\author{
E. Mackenney, D. Perea Tortosa, P. Hernández Ros

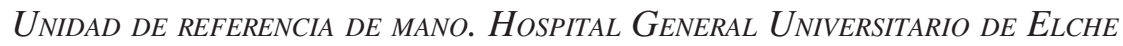 \\ Correspondencia: \\ emackenney@coma.es
}

\section{INTRODUCCIÓN}

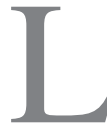

os nuevos conocimientos de la cicatrización y nutrición tendinosa y los progresos de la cirugía de la mano y de la microcirugía; así como, el perfeccionamiento de las técnicas de rehabilitación, han permitido mejorar los resultados. Sin embargo, a pesar de todo ello el tratamiento de las lesiones de los tendones flexores sigue siendo un problema difícil, que puede alterar el pronóstico funcional de la mano.

El conocimiento de la morfología, anatomía, aporte vascular, nutrición, características biomecánicas de los tendones y de los materiales de suturas y de los mecanismos de cicatrización, es esencial para el correcto tratamiento de las lesiones traumáticas de los tendones flexores de los dedos de la mano y para poder desarrollar nuevas técnicas quirúrgicas y pautas de rehabilitación, que permitan obtener mejores resultados.

\section{ANATOMÍA DE LOS TENDONES}

El tendón es en sí un 70\% de fibras longitudinales de colágeno esencialmente tipo I, aunque también contiene un 5 por ciento de tipo III y pequeñas cantidades de tipo $\mathrm{V}^{1-3}$, con pocas células, los tenocitos, fibroblastos cuyo papel es la producción de colágeno y la reorganización de la matriz extracelular. El tendón tiene una estructura jerárquica, incluyendo moléculas de colágeno, fibrillas, haces de fibrillas (fibras), fascículos y unidades tendinosas, que discurren paralelas al eje geométrico. En el tendón maduro, los haces de fibrillas de colágeno (fibras) tiene un diámetro entre 1 y $300 \mu \mathrm{m}$ y las fibrillas tienen un diámetro entre 20 a $280 \mathrm{~nm}^{1-3}$. Las moléculas de colágeno se combinan para formar unidades de microfibri- llas, subfibrillas y fibrillas. Estas unidades están ordenadas en haces estrechamente compactos, estrictamente paralelos, orientados en un patrón longitudinal específico (Figura 1). Las fibrillas se unen para formar fascículos, que se mantienen unidos por tejido conectivo laxo, el endotendón, que permite el movimiento longitudinal de los fascículos de colágeno y contiene vasos sanguíneos, linfáticos y nervios. Las fibrillas están en una matriz rica en proteoglicanos, glicoproteínas y agua. Una fina capa fibrosa, el epitenon, cubre la superficie externa del tendón lo que permite su deslizamiento en la vaina sinovial. Los tendones que no están en una vaina sinovial tienen una lámina de tejido conectivo laxo que los rodea: el paratenon $^{4}$ (Figura 2).

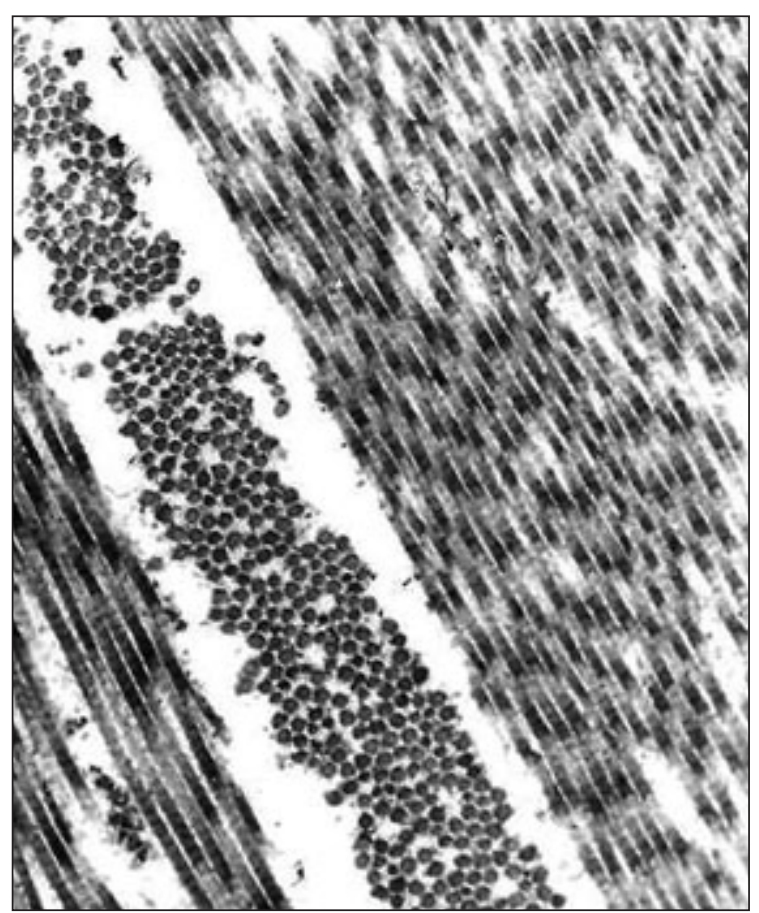

Figura 1: Fibras de colágeno. Microscopía electrónica de transmisión. X6000. 


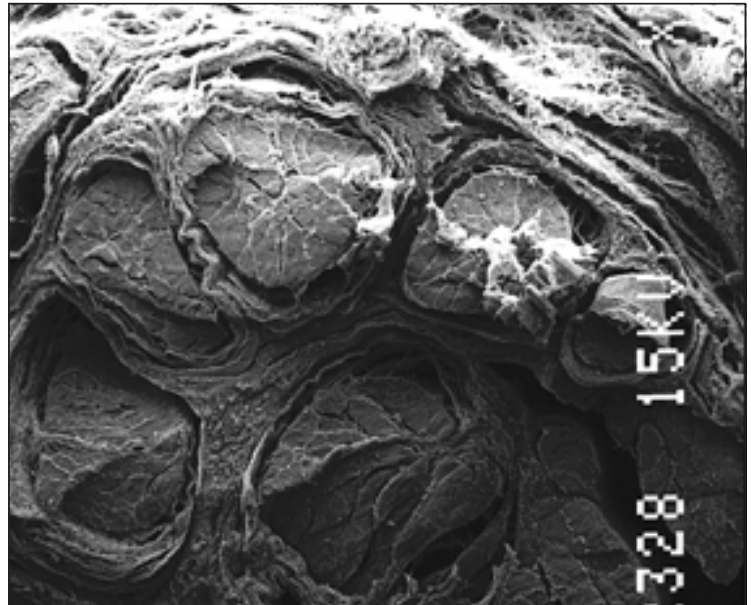

Figura 2: Tendones flexores en túnel del carpo. Microscopía electrónica de barrido.

\section{VAINAS SINOVIALES}

Los tendones flexores en los dedos están contenidos en un túnel fibro-óseo con segmentos de tejido fibroso de mayor densidad. En el lado de la superficie de fricción se origina un mesotendón de doble hoja y se une al epitendón que cubre la superficie del tendón. El deslizamiento del tendón se facilita por el líquido sinovial que sale de la membrana sinovial parietal y visceral o epitenon. La difusión de líquido sinovial aporta una alternativa nutricional efectiva, con una rápida difusión mediante un mecanismo de bombeo conocido como imbibición, en el cual el líquido pasa a los intersticios del tendón por pequeños conductos en su superficie cuando el tendón se flexiona y extiende ${ }^{5,6}$. El conocimiento de la disposición anatómica de la sinovia en la mano es también importante en los procesos infecciosos, aunque la incidencia de los mismos sea actualmente infrecuente ${ }^{7}$.

\section{SISTEMA DE POLEAS}

El sistema de poleas ${ }^{8}$ mantiene a los tendones en contacto con el esqueleto, dirigiendo el tendón, actuando como un transmisor mecánico y evitando el fenómeno de cuerda de violín. Por definición las poleas son bandas de tejido fibroso de grosor y anchura variable que recubre la vaina sinovial y se han identificado: la polea aponeurótica palmar, cinco poleas anulares (A1-A5) y tres poleas cruciformes (C1-C3). Las poleas A2 y A4 son las que más importancia biomecánica tienen, la pérdida de ellas disminuye la movilidad del dedo y su potencia y puede condicionar una contractura en flexión de las articulaciones interfalángicas ${ }^{9-12}$.

\section{VASCULARIZACIÓN TENDINOSA}

Los tendones reciben su aporte sanguíneo de los vasos del perimisio, de la inserción perióstica y del tejido circundante a través de vasos en el paratendón o mesotendón. En la vaina digital el tendón tiene un aporte vascular discontinuo a través de vinculas en la cara dorsal del tendón, dos para cada tendón, una corta y una larga ${ }^{5,15-17}$. Tanto el flexor digitorum superficialis como el profundus tienen áreas sin vascularización, y estos segmentos avasculares se nutren predominantemente por difusión, mientras que las partes vasculares la tienen por perfusión vascular. La difusión es más efectiva que la perfusión y puede, en ausencia de perfusión, nutrir a la mayoría de los tendones ${ }^{14}$. Fuera de la vaina digital la vascularización proviene del mesotendón, por tractos vasculares entre las fibras de colágeno.

\section{CICATRIZACIÓN TENDINOSA}

Los tendones tienen una capacidad de cicatrización extrínseca y una intrínseca, la primera dependerá de una proliferación conjuntiva vascular y fibroblastos. La cicatrización intrínseca tiene lugar por fibroblastos intra tendinosos y por nutrición a partir del líquido sinovial. Existe una primera fase inflamatoria, entre las 48 y 72 horas, seguida de una fase fibroblástica o formadora de colágeno, de 5 días a 4 semanas y una fase de remodelación hasta los 4 meses. Durante la fase inflamatoria la resistencia de la reparación depende completamente de la sutura, pero aumenta rápidamente durante la fase fibroblástica. Cuando la cicatrización extrínseca predomina, se forman también adherencias entre el tendón y los tejidos circundantes; mientras que cuando la cicatrización está basada en la actividad celular intrínseca habrá menos adherencias y de menor densidad ${ }^{4,5,18,19}$. 


\section{ANAMNESIS Y EXPLORACIÓN CLÍNICA}

El mecanismo lesional y las circunstancias del accidente deben definirse, con especial énfasis en la posición de los dedos en el momento de la lesión, ya que permite conocer una posible migración del cabo tendinoso proximal.

La exploración debe ser minuciosa, con un alto nivel de suspicacia. En heridas pequeñas, en ocasiones puntiformes, pueden lesionarse los tendones y los nervios colaterales. Se debe explorar sistemáticamente los tendones flexores superficialis y profundus, la sensibilidad y la vascularización.

\section{ZONAS TOPOGRÁFICAS O NIVEL DE LA LESIÓN TENDINOSA}

Verdan y Michon en $1961^{20}$ describieron siete zonas, que posteriormente fueron modificadas en un acuerdo del Comité de Lesiones Tendinosas de la Federación Internacional de Sociedades de Cirugía de la Mano a las cinco zonas presentadas por Eaton y Weilby ${ }^{21}$, cada una de ellas con sus características anatómicas propias, que condicionan una actitud terapéutica especial (Figura 3).

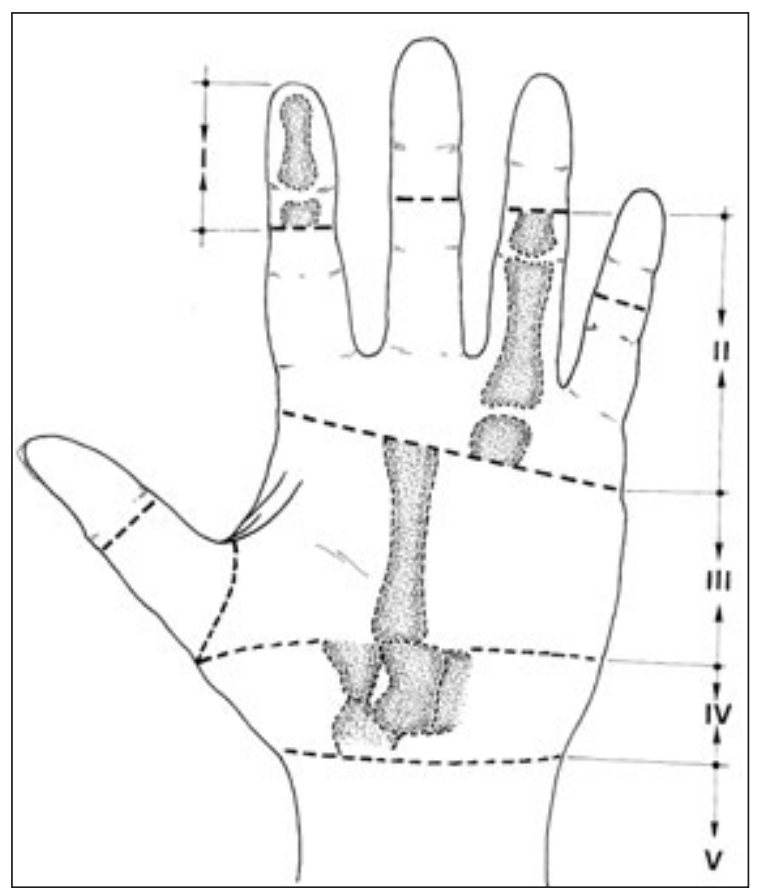

Figura 3: Clasificación en zonas de las lesiones de los tendones flexores en la mano.
Zona I, desde la base de la falange distal a la diáfisis de la falange media, con lesión del tendón flexor digitorum profundus distal a la inserción del flexor digitorum superficialis. El tendón puede presentar una alteración vascular por migración proximal, con afectación de las vinculas. El tendón puede afectarse junto a un fragmento óseo. En su reparación puede requerir una sutura o una reinserción ósea con un "pull-out" o un anclaje óseo.

Zona II, se extiende desde la diáfisis de la falange media hasta la metáfisis distal del metacarpiano. En ella pueden lesionarse uno o los dos tendones flexores y el sistema de poleas. Requerirá una cirugía cuidadosa, con sutura de ambos tendones, cuando ello sea posible y una reconstrucción de las poleas.

Zona III. Comprende la palma de la mano distal al ligamento anular del carpo hasta la metáfisis distal de los metacarpianos. Pueden lesionarse los dos tendones o sólo el superficial y también los nervios colaterales y los músculos lumbricales.

Zona IV, lesión tendinosa en el túnel del carpo, pudiéndose afectar ambos tendones o sólo el superficial y también el nervio mediano.

Zona V. Lesión en muñeca o antebrazo, con lesión tendinosa o musculotendinosa. Pueden lesionarse los tendones flexores de los dedos y pulgar, el flexor carpi radialis, el palmaris longus, los nervios mediano y/o nervio cubital, las arterias radial y/o cubital.

\section{TÉCNICA QUIRÚRGICA}

La cirugía de los tendones flexores debe ser cuidadosa, minuciosa y atraumática. Se debe disponer de todas las condiciones necesarias, con anestesia locorregional o general, isquemia con manguito neumático, instrumental de cirugía de mano y de microcirugía, gafa lupa y en determinadas circunstancias microscopio quirúrgico. El cirujano debe tener experiencia y la suficiente habilidad técnica.

La técnica de sutura tendinosa debe adquirirse en un quirófano experimental, con suturas in vitro. Los tendones flexores de patas de cerdo o de pollo son excelentes para ello y permiten adquirir el dominio de los diferentes tipos de sutura y de las técnicas de reconstrucción de poleas ${ }^{22,23}$. 


\section{OBJETIVOS DE LA SUTURA TENDINOSA}

La sutura de los tendones flexores debe cumplir varios objetivos: (1) anatómico, recuperar la congruencia tendinosa, (2) fisiológico, respetar la vascularización y la circulación sinovial y (3) biomecánico, solidez de la sutura.

Una sutura tendinosa ideal es aquella sutura realizada con facilidad, con nudos estables, con una unión suave de los cabos tendinosos, con una buena coaptación, con mínima interferencia con la vascularización y con la suficiente resistencia durante la cicatrización para permitir una movilización precoz.

\section{VÍAS DE ABORDAJE}

Los abordajes no deben comprometer la viabilidad de los colgajos cutáneos y no deben crear cicatrices retractiles. Las incisiones en zigzag de Brunner son las más utilizadas ${ }^{24}$.

\section{DETALLES TÉCNICOS}

La apertura de la vaina sinovial se realiza con un colgajo que pueda ser suturado posteriormente. Si el cabo proximal ha migrado proximal, debe ser recuperado de la forma más atraumática posible para no lesionar la vaina digital. Se han descrito diferentes métodos, como la expresión de proximal a distal con un Esmarch, el "milking" u ordeñado de proximal a distal con la muñeca y las articulaciones metacarpofalángicas en flexión, la utilización de catéteres de aspiración, sondas de silicona, sondas vesicales infantiles o pasatendones ${ }^{25,26}$.

\section{TÉCNICA DE SUTURA DE LOS TENDONES FLEXORES}

Diferentes autores han demostrado que la resistencia de la reparación de los tendones flexores es proporcional al número de hilos que cruzan la zona de reparación, siendo la de cuatro hilos el doble de resistente que la de dos y la seis tres veces más resistente ${ }^{27-29}$. Sin embargo, un gran número de hilos aumenta la complejidad de la técnica y la cantidad de material en la zona de la lesión e interfiere en la cicatrización; por ello, se debe buscar la sutura que reúna la mayor resistencia posible con la mínima cantidad de sutura, que deberá eso sí tener como mínimo cuatro hilos.

El tipo y calibre del material de sutura ha sido y es motivo de continua experimentación, intentando obtener la sutura con máxima resistencia y mínima reacción ${ }^{30}$. Si bien las suturas metálicas son las que mayor resistencia tienen su uso es complejo, por lo que generalmente se prefiere una sutura de polipropileno monofilamento (Prolene) o un poliéster trenzado (Ethibond), no reabsorbible, de 3/0 ó 4/0.

\section{SUTURA PRINCIPAL ("CORE SUTURE")}

La sutura de $\operatorname{Kessler}^{31}$ es la que más frecuentemente se ha utilizado y se sigue utilizando ${ }^{32}$, con sus posteriores modificaciones, Kessler modificado $^{33,34}$ y Tajima ${ }^{35}$. Sin embargo, es importante conocer las suturas clásicas, de Bunnell ${ }^{36}$, Masson-Allen ${ }^{37}$, Kleinert et al. ${ }^{38}$, Tsuge et al..$^{39}$, al igual que las nuevas suturas de cuatro o seis hilos, como las descritas por McLarney et al. ${ }^{40}$, Savage $^{27}$, Tanget al. ${ }^{41}$, Wagner et al. ${ }^{42}$, Winters et al. ${ }^{43}$; ya que del estudio de las mismas podemos determinar aquella que más de adapta a los requisitos imprescindibles (Figura 4).

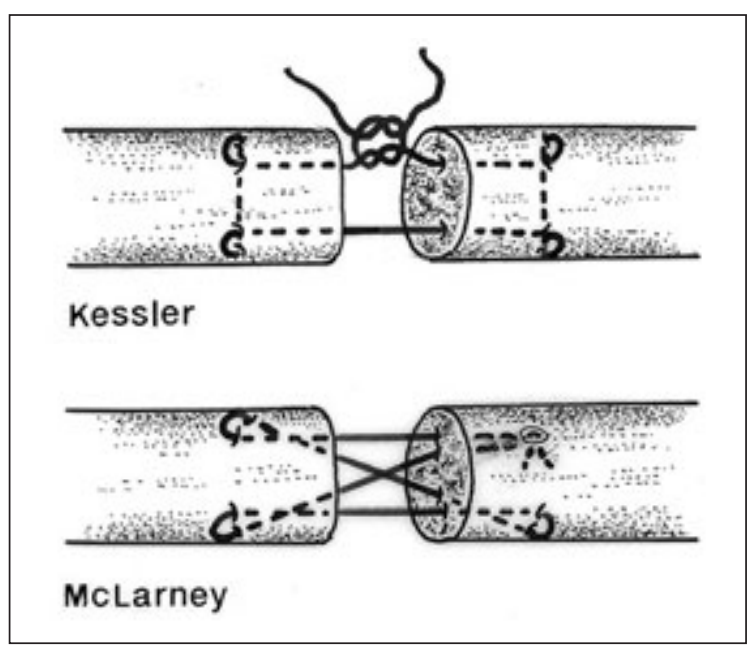

Figura 4: Sutura tendinosa principal. Sutura de Kessler ${ }^{33,34}$ modificada. Sutura cruciforme de McLarney et al. ${ }^{40}$. 
Los estudios comparativos muestran que las suturas cruciformes y las de seis hilos aumentan la resistencia de carga y previenen la separación de los borde de la sutura en relación a las de dos hilos.

\section{OTROS DETALLES TÉCNICOS DE IMPORTANCIA SON:}

1. La ubicación de la sutura en el plano palmar-dorsal, que clásicamente se ha considerado que debía ser en la mitad palmar del tendón, para no lesionar la vascularización intrínseca de los tendones ${ }^{44}$, se ha visto reconsiderada por el reconocimiento de que los tendones se nutren principalmente por difusión ${ }^{11,16}$ y de que existen zona avasculares en el tendón ${ }^{12,45}$ y por los estudios de Aoki et al. ${ }^{46}$ y de Soejima et al. ${ }^{47}$ que han demostrado que las suturas colocadas en la zona dorsal tienen una resistencia a la tracción significativamente mayor y una menor resistencia al deslizamiento del tendón, que si se colocan en la zona palmar.

2. La localización del nudo. Si se deja en el tendón en la zona de la lesión, puede disminuir la resistencia de la reparación ${ }^{46-48}$, aunque ello es así sólo durante las seis primeras semanas, y si hacemos el nudo fuera del tendón habrá una ventaja biomecánica inicial, pero la resistencia al deslizamiento es mayor y los nudos pueden atraparse en las poleas. Una alternativa es hacer una pequeña incisión longitudinal en la superficie del tendón y anudar el punto en ella, de tal manera que el nudo quede enterrado por debajo de la superficie del tendón.

3. El número de nudos. Una sutura con un nudo es potencialmente más fuerte que una con dos porque esta última se romperá en el nudo más débil de los dos. Más nudos significan que habrán más zonas potencialmente débiles ${ }^{49}$.

Un nudo con una triple lazada es lo indicado, la primera lazada con suficiente tensión para afrontar los extremos del tendón, la segunda en sentido inverso para bloquear la primera y una tercera lazada para asegurar las dos precedentes

4. La distancia del punto de sujeción a la zona de sección. Al desplazar el punto de agarre de la sutura a $10 \mathrm{~mm}$ de la zona de sección del tendón aumenta la resistencia a la tracción, ya que au- menta la interfase sutura-tendón y aporta un mayor poder de agarre en las fibras de colágeno ${ }^{50,51}$.

\section{SUTURAS EPITENDINOSAS O CIRCUNFERENCIALES}

Las suturas epitendinosas aumentan la resistencia de tracción de la reparación tendinosa, aportando un refuerzo a la resistencia de la reparación de un $35 \%$ en las secciones transversales y de un $11 \%$ en las secciones oblicuas ${ }^{50}$, y también una reducción significativa a la separación entre los extremos tendinosos ${ }^{34,48,52,53}$. Diferentes tipos han sido descritos, circunferenciales simples, simples bloqueada, bloqueada de Linn et al. ${ }^{54} \mathrm{o}$ las horizontales como las suturas de Halsted o las de Mashadi y Amis ${ }^{55}$ (Figura 6). Estudios comparativos de ellas ${ }^{56,57}$, muestran que la sutura bloqueada de Lin et al. ${ }^{54}$ es la que mayor poder de sujeción tiene, pero también la de mayor fricción al deslizamiento y es la más difícil técnicamente (Figura 5).

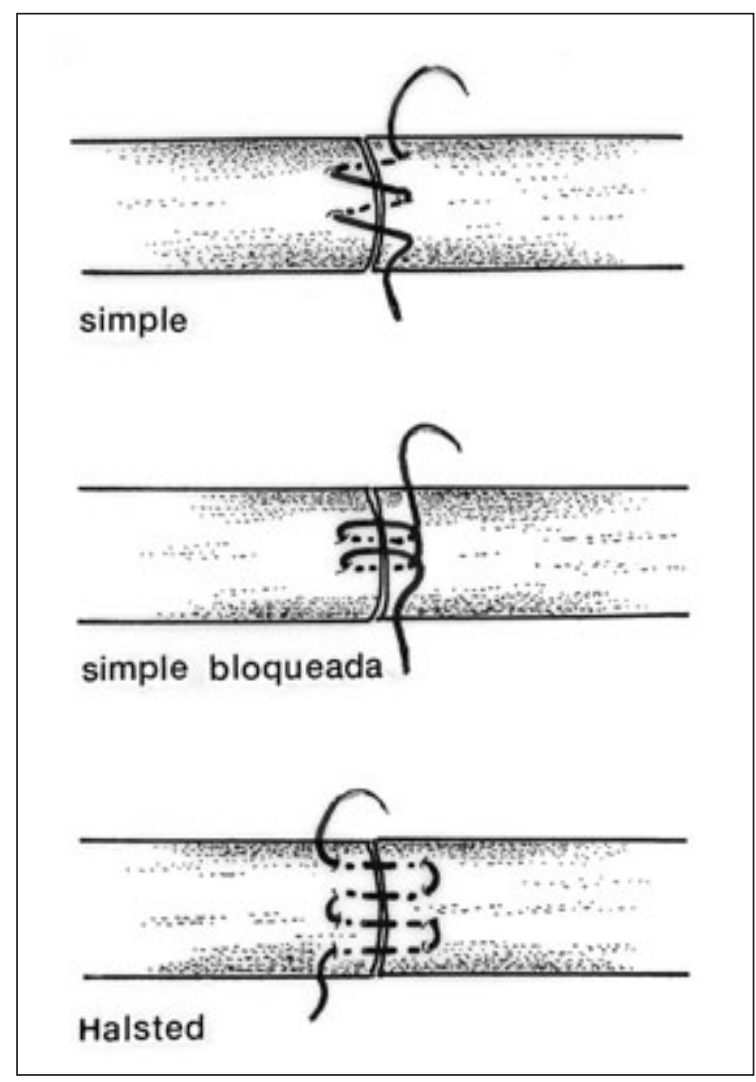

Figura 5: Suturas circunferenciales. Simple, simple bloqueada y sutura de Halsted. 
Un detalle técnico, complementario al tipo de sutura a realizar, es el de la distancia del punto de sujeción a la zona de sección, que se aconseja sea de $2 \mathrm{~mm}^{58}$ ó $4 \mathrm{~mm}{ }^{59}$, ya que ello confiere una mayor resistencia que si sólo es de $1 \mathrm{~mm}$.

\section{SUTURA DE ELECCIÓN}

La sutura de cruciforme de McLarney et al. ${ }^{40}$ es quizás una sutura de elección, ya que tiene la resistencia de una reparación de cuatro hilos y la sencillez y los requisitos de manipulación de los cabos tendinosos de una reparación de dos hilos; complementada con una sutura circunferencial bloqueada, y siguiendo todos los detalles técnicos descritos (Figura 6).
El objetivo final de la sutura tendinosa es permitir una movilización precoz, con una prevención de adherencias, promoviendo el deslizamiento y la diferenciación de los tejidos blandos y restaurando la función.

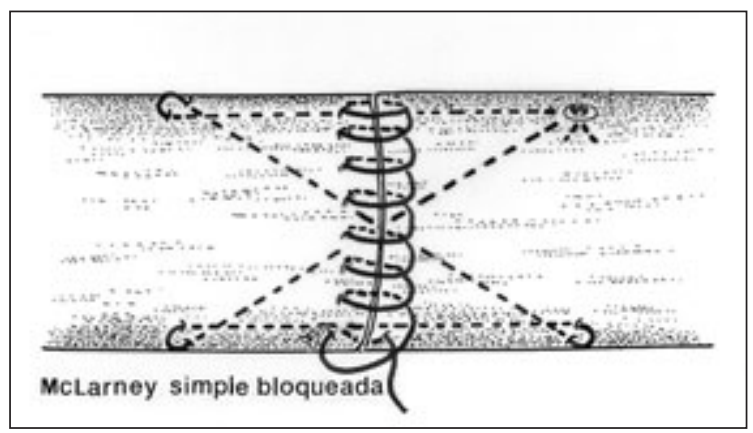

Figura 6: Sutura tendinosa de McLarney ${ }^{40}$ con punto intratendinoso y sutura circunferencial simple bloqueada.

\section{BIBLIOGRAFÍA}

1. Woo SL, An K, Arnoczky SP, et al. Anatomía, biología y biomecánica de los tendones, ligamentos y meniscos. En: Ciencias Básicas en Ortopedia. American Academy of Orthopaedic Surgeons. Rosemont. IL, 1997: 49-91.

2. Silver FH, Freeman JW, Bradica G. Structure and function of ligaments, tendons, and joint capsule. En: Walsh WR (Ed). Orthopaedic Biology and Medicine. Repair and regeneration of ligaments, tendons and joint capsule. New Jersey: Humana Press, 2006: 15-47.

3. Riechert K, Labs K, Lindenyhayn K, et al. Semiquantitative analysis of types I and III collagen form tendons and ligaments in a rabbit model. $J$ Orthop Sci, 2001; 6: 68-74.

4. Stephens PC, Dona E, Niechoda B, et al. Tendons of the hand. Anatomy, repair, healing and rehabilitation. Repair and regeneration of ligaments, tendons and joint capsule. En: Walsh WR (ED). Orthopaedic Biology and Medicine. New Jersey: Humana Press, 2006: 49-86.

5. Schneider LH. Flexor tendon injuries. En: Anatomy and function of the flexor tendon system. Boston/Toronto: Little, Brown and Company, 1985; 15-8.

6. Idler RS. Anatomy and biomechanics of the digital flexor tendons. Hand Clinics, 1985; 1: 3-11.

7. Kanavel AB. The tendon sheaths: A discussion of their anatomical distribution and relations, with surgical deductions. En: Infection of the Hand. $5^{\text {th }}$. Philadelphia: Ed Lea and Febiger, 1925: 100-10.

8. Doyle JR, Blythe W. The finger flexor tendon sheath and pulleys: anatomy and reconstruction. En: AAOS Symposium on Tendon Surgery in the Hand. St Louis: CV Mosby Co, 1975: 81-7.

9. Doyle JR. Anatomy of the flexor tendon sheath and pulley system: A current review. J Hand Surg Am, 1989; 14: 349-51.

10. Doyle JR. Palmar and digital flexor tendon pulleys. Clin Orthop, 2011; 383: 84-96.

11. Amis AA, Jones MM. The interior of the flexor tendon sheath of the finger. J Hand Surg $\mathrm{Br}$, 1988; 70: 583-87.

12. Lundborg G, Myrhage R. The vascularization and structure of the human digital tendon sheath as related to flexor tendon function. Scan J Plast Reconst Surg, 1977; 11: 195 -203.

13. Manske PR, Lesker PA. Palmar aponeurosis pulley. J Hand Surg Am, 1983; 8: 259-63.

14. Strickland JW. Development of flexor tendon surgery: Twentyfive years of progress. J Hand Surg Am, 2000; 25: 214-35.

15. Leffert RD, Weiss $C$, Athanasoulis CA. The vincula. J Bone Joint Sur Am, 1974; 56: 1191-98.

16. Manske PR, Lesker PA. Flexor tendon nutrition. Hand Clinics, 1985; 1: 13-24.

17. Zbrodowski A, Gajisin S, Grodecki J. Mesotendons of digital flexor muscles and their vasculature. The Hand, 1981; 13: 231- 38.

18. Manske PR, Gelberman $\mathrm{RH}$, Lesker PA. Flexor tendon healing. Hand Clinics, 1985; 1: 2534.

19. Woo SLY, An Kai-Nan, Arnoczky SP, et al. Anatomy, biology, and biomechanics of tendon, ligament, and meniscus. En: Orthopaedic Basic Science. American Academy of Orthopedic Surgery. Rosemont. IL, 1994, 45-87.

20. Verdan C, Michon J. Le traitment des plaies des tendons flechis- 
seurs des doigts. Rev Chir Orthop, 1961; 47: 285-425.

21. Kleinert HE, Verdan C. Report of the Committee on Tendon Injuries. Internacional Federation of Societies for Surgery of the Hand. J Hand Surg Am, 1983; 8: 794-8.

22. Smith AM, Forder SR, Annapuredy SR, et al. The porcine forelimb as a model for human flexor tendon surgery. J Hand Surg Eur, 2005; 30: 307-9.

23.Tan S, Power D, Rajaratnam V. Animal models for training in tendon surgery. J Hand Surg Eur, 2010; 35: 152-3.

24. Brunner JM. The zig-zag volardigital incision for flexor-tendon surgery. Plast Reconstr Surg, 1967; 40: 571-4.

25. Coats RW, Echevarria-Oré JC, Mass DP. Acute flexor tendon repair in zone II. Hand Clinics, 2005; 21: 173-9.

26. Sourmelis SG McGrouther DA. Retrieval of the retracted flexor tendon. J Hand Surg Br, 1997; 12: 109-11.

27. Savage R. In vitro studies of a new method of flexor tendon repair. J Hand Surg Br, 1985; 10: 135-41.

28. Savage R, Risitano G. Flexor tendon repair using a "six strand" method of repair and early active mobilization. J Hand Surg Br, 1989; 14: 396-9.

29. Winters SC, Gelberman $\mathrm{RH}$, Woo SLY, et al. The effects of multiple-strand suture methods on the strength and excursion of repaired intrasynovial flexor tendons: a biochemical study in dogs. J Hand Surg Am, 1992; 17: 89-91.

30. Trail IA, Powell ES, Noble J. The mechanical strength of various suture techniques. J Hand Surg Br, 1992; 17: 89-91.

31. Kessler I. The grasping technique for tendon repair. The Hand, 1973; 5: 253-5.

32. Healy C, Mulhall KJ, BouchierHayes DJ, et al. Practice patterns in flexor tendon repair. Ir J Med Sci, 2007, 176: 41-4.

33. Kubota H, Miyanishi K, Hoshino $\mathrm{S}$. Effect of a new repair techni- que on biomechanical properties of canine flexor tendons. Hand Surg, 1998; 3: 247-55.

34. Wada A, Kubota $H$, Hatanaka $\mathrm{H}$, et al. The mechanical properties of locking and grasping suture loop configuration in four-strand core suture techniques. J Hand Surg Eur, 2000; 25: 548-51.

35. Tajima T. History, current status and aspects of hand surgery in Japan. Clin Orthop, 1984; 184: 41-9.

36. Bunnell S. Repair of nerves and tendons of the hand. $\mathrm{J}$ Bone Joint Surg, 1928; 10: 1-26.

37. Masson ML, Allen HS. The rate of healing of tendons. Ann Surg, 1941; 113: 424-59.

38. Kleinert HE, Kutz JE, Atasoy E, et al. Primary repair of flexor tendons. Orthop Clin North Am, 1973; 4: 865-76.

39. Tsuge K, Ikuta $Y$, Matshuishi $Y$. Intra-tendinous tendon suture in the hand. A new technique. Hand, 1975; 7: 250-5.

40. McLarney E, Hoffman $\mathrm{H}$, Wolfe SW. Biomechanical analysis of the cruciate four-strand flexor tendon repair. J Hand Surg Am, 1999; 24: 295-301.

41. Tang JB, Wang B, ChenF, et al. Biomechanical evaluation of flexor tendon repair techniques. Clin Orthop, 2001; 386: 252-9.

42. Wagner WF Jr, Carrol C IV, Strickland JW, et al. A biomechanical comparison of techniques of flexor tendon repair. J Hand Surg Am, 1994; 19: 979-83.

43. Winters SC, Gelberman $\mathrm{RH}$, Woo SLY, et al. The effects of multiple strand suture methods on the strength and excursion of repaired intrasynovial flexor tendons flexor tendons: a biomechanical study in dogs. J Hand Surg Am, 1988; 23: 97-104.

44. Caplan HS, Hunter JM, Merkin RJ. Intrinsic vascularization of flexor tendons. En: AAOS Symposium on Tendon Surgery in the Hand. St Louis: CV Mosby Co, 1975: 48-58.

45. Ochiai N, Matsui T, Miyaji N, et al. Vascular anatomy of flexor tendos. Vincular system and blood supply of the profundus tendon in the digital sheath. J Hand Surg Am, 1979; 4: 32130.

46. Aoki M, Manske PR, Pruitt DL, et al. Work of flexion after flexor tendon repair according to the placement of sutures. Clin Orthop, 1995; 320: 205-10.

47. Soejima O, Diao E, Lotz JC, et al. Comparative mechanical analysis of dorsal versus palmar placement of core suture for flexor tendon repairs. J Hand Surg Am, 1995; 20: 8017.

48. Pruitt DI, Aoki M, Manske PR. Effect of suture knot location on tensile strength after flexor repair. J Hand Surg Am, 1996; 21: 969-73.

49. Aoki M, Pruitt DL, Kubota H, et al. Effect of suture knots on tensile strength of repaired canine flexor tendons. J Hand Surg Br, 1995; 20: 72-5.

50. Yuen MH, IP WY. Tensile strength of modified 4-strand cruciate technique for transversely or obliquely lacerated tendons. J Orthop Surg, 2007: 15: 27-31.

51. Lee SK, Goldstein RY, Zingman $A$, et al. The effects of core suture purchase on the biomechanical characteristic of a multistrand locking flexor tendon repair: a cadaveric study. J Hand Surg Am, 2010; 35: 1165-71.

52. Diao E, Hariharan JS, Soejima $O$, et al. Effect of peripheral suture depth on strength repair. J Hand Sur Am, 1996; 21: 23439.

53. Silfverskiöld, May EJ, Törnvall $\mathrm{AH}$. Gap formation during controlled motion after tendon repair in zone II: A prospective clinical study. J Hand Surg Am, 1992; 17: 539-46.

54. Linn GT, An KN, Amadio PC, et al. Biomechanical studies of running suture for flexor tendon repair in dogs. J Hand Surg Am, 1988: 553-8.

55. Mashadi ZB, Amis AA. Strenght of the suture in the epitenon 
and within the tendon fibres: development of stronger peripheral suture technique. $\mathrm{J}$ Hand Surg Br, 1992: 171-5.

56. Kubota H, Aoki M, Pruitt DL, et al. Mechanical properties of various circumferencial tendon sutures technique. J Hand Surg Eur, 1996: 474-80.
57. Dona E, Turner AWL, Gianoutsos MP, et al. Biomechanical properties of four circumferencial flexor tendon suture techniques. J Hand Surg Am, 2003; 28: 824-31.

58. Merrell GA, Wolfe SW, Kacena $W J$, et al. The effect of increased peripheral suture purchase on the strength of flexor tendon repairs. J Hand Surg Am, 2003; 28: 464-8.

59. Hirpara KM, Sullivan PJ, O'Neill $B$, et al. The optimum length of the Silfverskiöld circumferencial cross stich. J Hand Surg Eur, 2009; 34: 651-5. 